\title{
Quantification of pleural effusion on CT by simple measurement
}

\author{
Martin Hazlinger ${ }^{\mathrm{a}}$, Filip Ctvrtlika ${ }^{\mathrm{a}}$ Katerina Langova ${ }^{\mathrm{b}}$, Miroslav Herman ${ }^{\mathrm{a}}$
}

\begin{abstract}
Aims. To find the simplest method for quantifying pleural effusion volume from CT scans.
Methods. Seventy pleural effusions found on chest CT examination in 50 consecutive adult patients with the presence of free pleural effusion were included. The volume of pleural effusion was calculated from a three-dimensional reconstruction of CT scans. Planar measurements were made on CT scans and their two-dimensional reconstructions in the sagittal plane and at three levels on transversal scans. Individual planar measurements were statistically compared with the detected volume of pleural effusion. Regression equations, averaged absolute difference between observed and predicted values and determination coefficients were found for all measurements and their combinations. A tabular expression of the best single planar measurement was created.

Results. The most accurate correlation between the volume and a single planar measurement was found in the dimension measured perpendicular to the parietal pleura on transversal scan with the greatest depth of effusion. Conversion of this measurement to the appropriate volume is possible by regression equation:

Volume $=0.365 \times b^{3}-4.529 \times b^{2}+159.723 \times b-88.377$.

Conclusion. We devised a simple method of conversion of a single planar measurement on CT scan to the volume of pleural effusion. The tabular expression of our equation can be easily and effectively used in routine practice.
\end{abstract}

Key words: $\mathrm{CT}$, chest, pleura, effusion, volume

Received: November 2, 2011; Accepted: April 5, 2012; Available online: June 1, 2012 http://dx.doi.org/10.5507/bp.2012.042

${ }^{a}$ Department of Radiology, University Hospital Olomouc and Faculty of Medicine and Dentistry, Palacky University Olomouc, Czech Republic ${ }^{b}$ Department of Medical Biophysics, Faculty of Medicine and Dentistry, Institute of Molecular and Translational Medicine, Palacky University Olomouc

Corresponding author: Martin Hazlinger, e-mail: Martin.Hazlinger@fnol.cz

\section{INTRODUCTION}

Pleural fluid is physiologically present in the pleural cavity of humans ${ }^{1,2}$. In normal conditions, its mean unilateral volume is $8.4 \pm 4.3 \mathrm{~mL}$ (ref. ${ }^{3}$ ). Increasing fluid amount increases lung capillary pressure and reduces lung compliance, which leads to difficult breathing and patient dyspnoea ${ }^{4}$. Pleurocentesis provides relief from dyspnoea; therefore, in addition to the clinical condition, quantification of pleural effusion amount is of major importance for patient management.

There are various ways of detecting and monitoring pleural effusion. The first and typical method is posteroanterior chest radiograph. Pleural effusion is visible on the posteroanterior projection as a meniscus at about $200 \mathrm{~mL}$ and will obliterate the hemidiaphragm at approximately $500 \mathrm{~mL}$ (ref. ${ }^{5}$ ). Effusions lower than $200 \mathrm{~mL}$ are detectable only when a lateral chest radiograph is taken pleural effusion becomes visible at approximately $50 \mathrm{~mL}$ as a meniscus in the posterior costophrenic sulcus 5 .

The method of choice for detecting pleural effusion in supine patients, particularly in intensive care unit patients, is sonography of pleural space ${ }^{6}$. Pleural effusion volume quantification is possible by the equation $y=47.6 \times x-$ 837 , where $y$ is the predicted effusion volume in millilitres and $x$ is the sonographically measured maximal thickness of the effusion lamella in millimetres ${ }^{6}$.
Computed tomography (CT) is the most proper method for detection and evaluation of pleural effusion. On CT scans, the effusion dimensions can be measured easily, but effusion volume determination is difficult. It can be calculated by automatic quantification using special software $^{7}$; however, this is not widely available. Mergo et al. ${ }^{8}$ described the quantification of pleural effusion amount from CT, but their calculation requires measurements in three planes.

In our pilot study ${ }^{9}$, we confirmed the possibility of using a single measurement on transversal CT scans to assess pleural effusion volume with reasonable accuracy. Consequently, the original group was extended and the final results are presented here.

\section{MATERIAL AND METHODS}

\section{Patient population}

Inclusion criteria: All contrast-enhanced CT examinations of the chest with the presence of free pleural effusion in patients older than 18 years. This age limit was chosen because of better concordance of size of the chest (no chest size dependence was considered). Examinations with intravenous contrast medium were used for a better discrimination between pleural effusion and the other structures, especially lung parenchyma. 


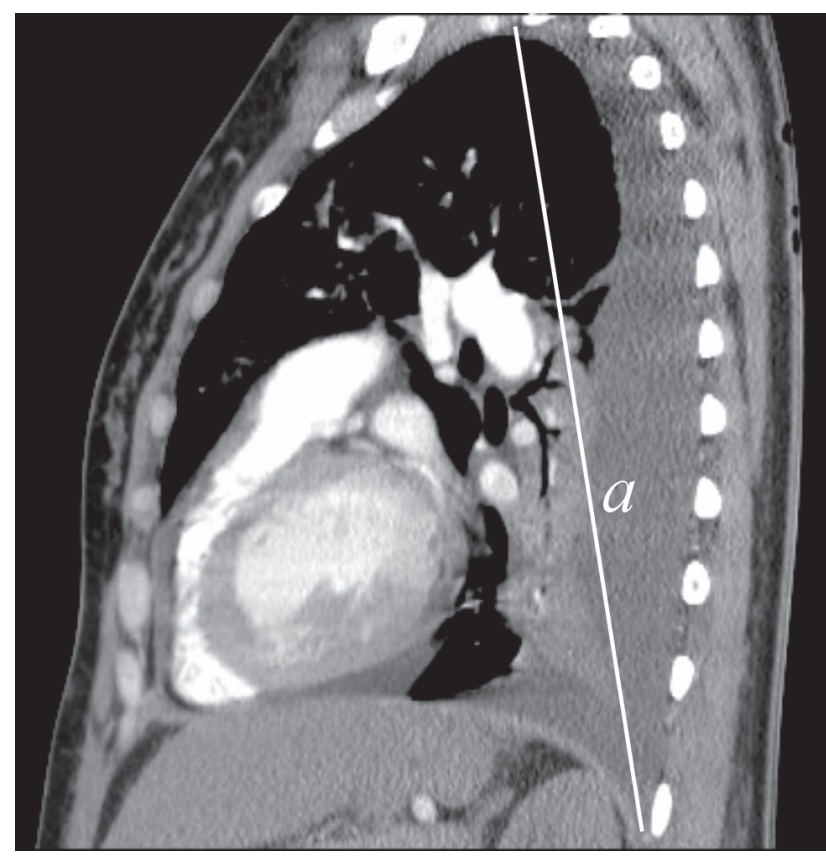

Fig. 1. The measurement technique of dimension $a$ on the sagittal CT scans reconstruction

Exclusion criteria: Patients with obvious pleural adhesion, empyema, pleural cavity bleeding, inserted pleural cavity drain and severe chest asymmetry.

From November 2009 to May 2010, 70 pleural effusions meeting the stated criteria were detected in 50 consecutive patients. There were 29 men and 21 women (mean age 65.5 years; age range, 24-87 years). Unilateral (22 right-sided, 8 left-sided) and bilateral pleural effusions were found in 30 and 20 patients, respectively.

\section{Measurement method}

CT examinations were performed by spiral technique on LightSpeed VCT XT (General Electric, Milwaukee, USA) machine with 64 detector rows. Examinations were primarily carried out with various slice thickness, but for further processing 5-mm scans were reconstructed in all cases.

The effusion volume was assessed on Advantage Windows 4.4 (General Electric, Milwaukee, USA) console by using the application Quick Paint. The area of pleural effusion was marked on each 5-mm scan. After processing all the scans, a three-dimensional reconstruction of pleural effusion was obtained. Its visual evaluation allowed us to verify the accuracy of measurement. When significant irregularities were found, the measurement was repeated. The software then calculated pleural effusion volume in millilitres. The effusion volume detected was in the range from $36 \mathrm{~mL}$ to $2990 \mathrm{~mL}$.

Subsequently, the effusion dimensions were measured on the same console at different levels on transversal scans and on sagittal reformation to find the most accurate measurement method. On reformatted images in the sagittal plane, the greatest craniocaudal effusion dimension was found - dimension $a$ (effusion height)

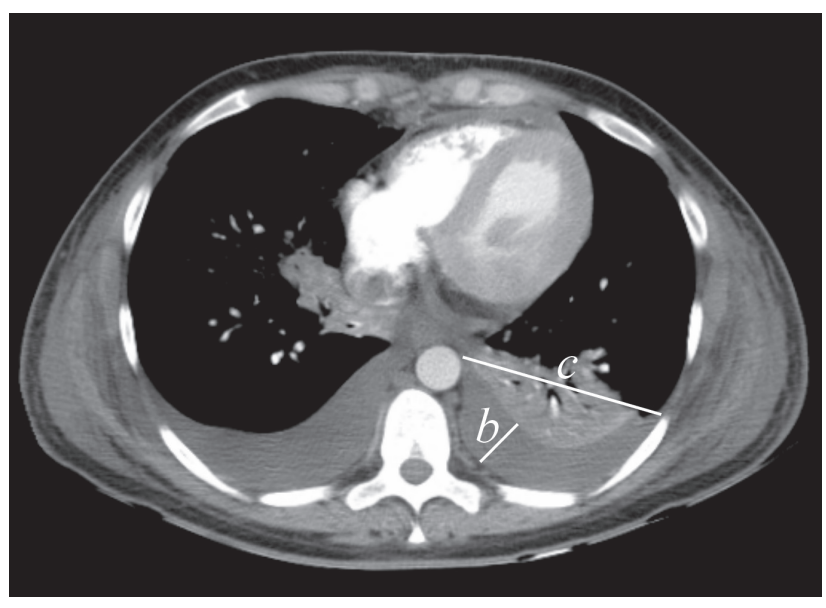

Fig. 2. The measurement technique of dimensions $b$ and $c$

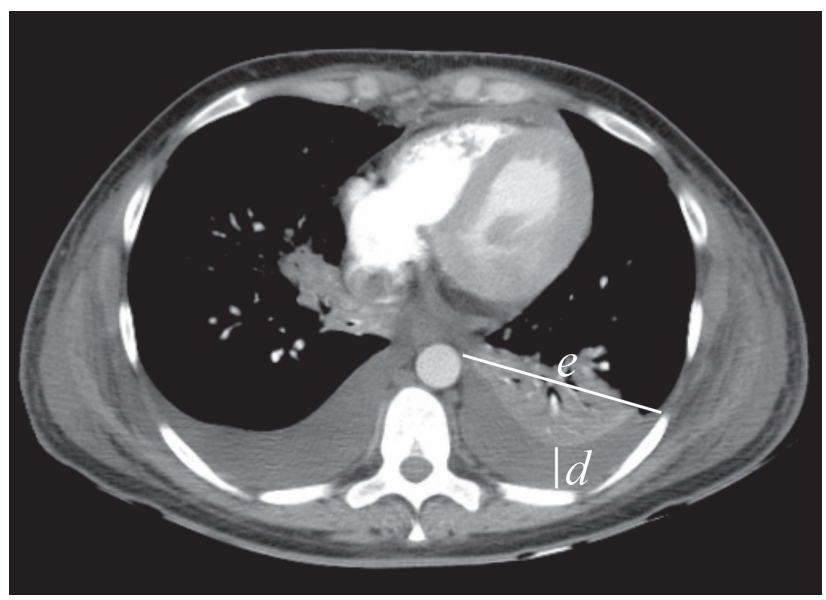

Fig. 3. The measurement technique of dimensions $d$ and $e$

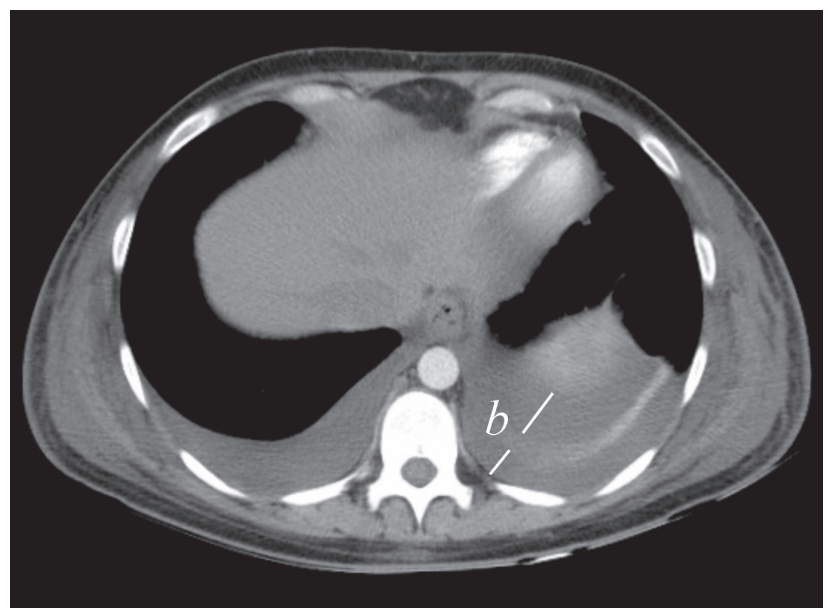

Fig. 4. The measurement technique of dimension $b$ when the diaphragm or lung interrupts the measurement line on CT scan

(Fig. 1). Dimension $b$ (effusion depth) was measured perpendicular to the parietal pleura on transversal scan where the greatest depth was found by scrolling all the images. Effusion width - dimension $c$ was measured on 
the same scan as the greatest distance between the most medial and lateral points of effusion (Fig. 2). The next measurement was performed ventrodorsally from the lowest spot of the effusion on transversal scan where it was found to be the largest by scrolling the images - dimension $d$. Effusion width - dimension $e$ was measured on the same scan as described above (Fig. 3). Afterwards, the same measurement method as in dimensions $b$ and $c$ was performed on the scan where the top of the appropriate hemidiaphragm was imaged - dimensions $f, g$, and on the scan $3 \mathrm{~cm}$ above - dimensions $h$, $i$. If the maximal effusion depth was interrupted by any structure (e.g. the diaphragm, lung, etc.) in any of the three measurement methods, this maximal depth was measured by omitting this structure (Fig. 4).

\section{Statistical analysis}

The strength of fit of statistical models was measured by determination coefficient. Regression equations were found for all measurements and their combinations. The data were analyzed by statistical software SPSS version 15 (SPSS Inc., Chicago, USA). The best method of single measurement was compiled in the synoptic and simple table calculated according to its regression equation.

\section{RESULTS}

The comparison of the dependence of individual planar measurements and their combinations on the pleural

Table 1. Determination coefficients for functionality: volume - combination of measurements. (Relation is statistically more significant when the number approaches 1.0. The best correlation is marked bold.).

\begin{tabular}{lcccccc}
\hline Measurement combinations & $a \times b \times b$ & $a \times b \times c$ & $a \times d \times d$ & $a \times d \times e$ & $a \times f \times g$ & $a \times h \times i$ \\
\hline Determination coefficient & 0.920 & 0.951 & 0.899 & $\mathbf{0 . 9 5 4}$ & 0.950 & 0.873 \\
Significance & $<0.0001$ & $<0.0001$ & $<0.0001$ & $<0.0001$ & $<0.0001$ & $<0.0001$ \\
\hline
\end{tabular}

Table 2. Determination coefficients for functionality: volume - single measurement. (Relation is statistically more significant when the number approaches 1.0. The best correlation is marked bold.)

\begin{tabular}{lccccc}
\hline Dimension & $a$ & $b$ & $d$ & $f$ & $h$ \\
\hline Determination coefficient & 0.633 & $\mathbf{0 . 9 4 9}$ & 0.940 & 0.903 & 0.718 \\
Significance & $<0.0001$ & $<0.0001$ & $<0.0001$ & $<0.0001$ & $<0.0001$ \\
\hline
\end{tabular}

Table 3. Regression equations for volume quantification based on the measurements performed and the average absolute variations calculated.

\begin{tabular}{llr}
\hline $\begin{array}{l}\text { Dimension / } \\
\text { dimension combi- } \\
\text { nations }\end{array}$ & Regression equations & $\begin{array}{r}\text { Average } \\
\text { variations }\end{array}$ \\
\hline$a$ & Volume $=0.241 \times a^{2.398}$ & $195.6 \mathrm{~mL}$ \\
$b$ & Volume $=0.365 \times b^{3}-4.529 \times b^{2}+159.723 \times b-88.377$ & $83.4 \mathrm{~mL}$ \\
$d$ & Volume $=0.396 \times d^{3}-6.17 \times d^{2}+177.012 \times d-87.775$ & $90.1 \mathrm{~mL}$ \\
$f$ & Volume $=0.516 \times f^{3}-6.7 \times f^{2}+150.763 \times f-10.541$ & $98.4 \mathrm{~mL}$ \\
$h$ & Volume $=191.99 \times \mathrm{h}-57.926$ & $136.3 \mathrm{~mL}$ \\
$a \times b \times b$ & Volume $=-3.4 \times 10^{-5} \times(a \times b \times b)^{2}+0.624 \times(a \times b \times b)+179.506$ & $193.2 \mathrm{~mL}$ \\
$a \times b \times c$ & Volume $=7.88 \times 10^{-9} \times(a \times b \times c)^{3}-6.0 \times 10^{-5} \times(a \times b \times c)^{2}+0.517 \times(a \times b \times c)+32.044$ & $71.0 \mathrm{~mL}$ \\
$a \times d \times d$ & Volume $=-3.9 \times 10^{-5} \times(a \times d \times d)^{2}+0.657 \times(a \times b \times c)+192.068$ & $208.2 \mathrm{~mL}$ \\
$a \times d \times e$ & Volume $=8.62 \times 10^{-9} \times(a \times d \times e)^{3}-7.1 \times 10^{-5} \times(a \times d \times e)^{2}+0.557 \times(a \times d \times e)+32.582$ & $68.0 \mathrm{~mL}$ \\
$a \times f \times g$ & Volume $=0.423 \times(a \times f \times g)+86.518$ & $82.0 \mathrm{~mL}$ \\
$a \times h \times i$ & Volume $=7.03 \times 10^{-8} \times(a \times h \times i)^{3}-2.481 \times 10^{-4} \times(a \times h \times i)^{2}+0.761 \times(a \times h \times i)+38.514$ & $93.2 \mathrm{~mL}$ \\
\hline
\end{tabular}


Table 4. Conversion of a single planar measurement $b$ to pleural effusion volume

\begin{tabular}{cc}
\hline $\begin{array}{c}\text { Dimension } b \\
(\mathrm{~cm})\end{array}$ & $\begin{array}{c}\text { Pleural effusion volume } \\
(\mathrm{mL})\end{array}$ \\
\hline 1 & 67 \\
1.5 & 142 \\
2 & 216 \\
2.5 & 288 \\
3 & 360 \\
3.5 & 431 \\
4 & 501 \\
4.5 & 572 \\
5 & 643 \\
5.5 & 714 \\
6 & 786 \\
6.5 & 859 \\
7 & 933 \\
7.5 & 1009 \\
8 & 1086 \\
8.5 & 1166 \\
9 & 1248 \\
9.5 & 1333 \\
10 & 1421 \\
\hline
\end{tabular}

effusion volume is shown in Tables 1 and 2. Regression equations for volume quantification based on the measurements performed and the average absolute variations calculated are shown in Table 3.

The most accurate correlation was found in combination $a \times d \times e$, where the determination coefficient was 0.954 . The best correlation for a single planar measurement was found for dimension $b$ with a determination coefficient of 0.949 . The results of all measurements and their combinations showed very good correlation and were highly significant $(<0.0001)$.

The equation for volume quantification based on dimension $b$ is defined by cubic dependence: Volume $=$ $0.365 \times b^{3}-4.529 \times b^{2}+159.723 \times b-88.377$. Its graphic illustration is in Fig. 5 and tabular expression in Table 4. The averaged absolute difference between observed and predicted values by using the stated formula is $\pm 83.4 \mathrm{~mL}$.

The determination coefficients for dimension $b$ calculated for right-sided and left-sided effusions were 0.96 and 0.865 , respectively. Both results are highly significant $(<0.0001)$.

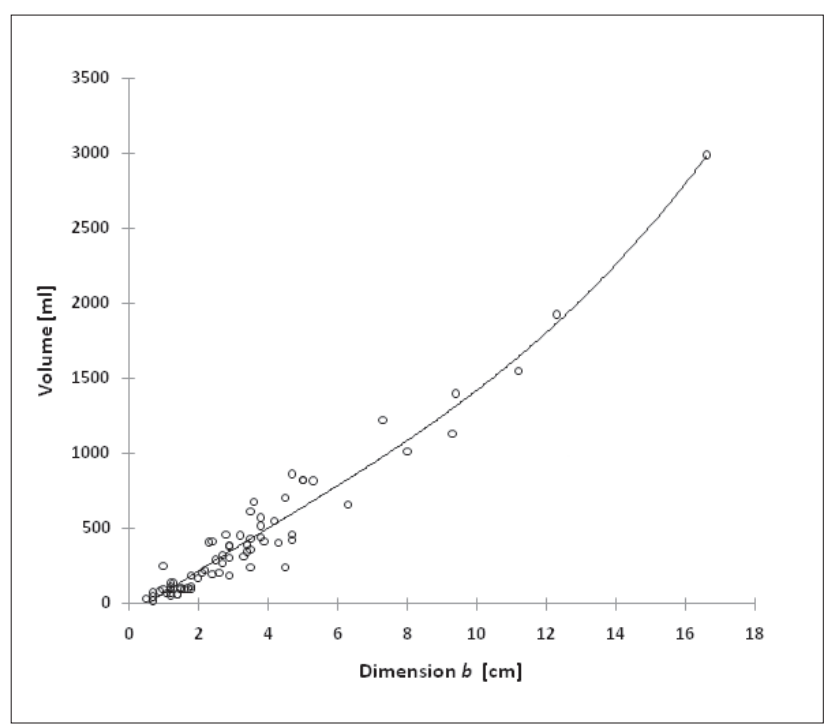

Fig. 5. The functionality graph of pleural effusion volume and dimension $b$

\section{DISCUSSION}

All the measurements and their combinations made, showed highly significant dependence on the volume ascertained and are usablein clinical practice. The most accurate and simplest quantification of pleural effusion volume by only one measurement was found to be the dimension perpendicular to the parietal pleura on CT scan with the greatest effusion depth (dimension $b$ ) with a determination coefficient of 0.949 and average absolute variation of $\pm 83.4 \mathrm{~mL}$. This measurement fulfilled our aim and a simple tabular expression (Table 4) can be used in everyday clinical practice. It can be stated, after slight rounding, that the greatest effusion depths of 2, 4, 6, 8, and $10 \mathrm{~cm}$ represent volumes of 200, 500, 800, 1100, and $1400 \mathrm{~mL}$ of pleural effusion, respectively.

The definitive results presented in this article are slightly different from those of our pilot study ${ }^{9}$. Even though the pilot data were included, the final result after increase of number of patients showed that the most accurate measurement is the maximal dimension perpendicular to the parietal pleura (dimension $b$ ), whereas the best results in the pilot study were obtained by using the maximal ventrodorsal measurement from the lowest spot of the effusion on transversal scan (dimension $d$ ). The difference between the measurement methods was small - up to $50 \mathrm{~mL}$. Both can be used in clinical practice.

Although we did not consider the dependence of our measurements on the chest size based on individual patient body habitus or gender, which would improve the final results, the statistical significance is high and the average absolute variation of $\pm 83.4 \mathrm{~mL}$ is small. There was also a slight difference in accuracy between the left and right side, with lower measurement precision on the left side, which is probably due to the presence of the heart. In spite of these variations, the method described is universal and only one simple table for conversion of a single planar 
measurement to pleural effusion volume need be used in everyday clinical practice (Table 4).

To our knowledge, only two descriptions of pleural effusion volume quantification from CT have been reported in the literature. Kalokairinos et al. ${ }^{7}$ based their method of pleural effusion volume quantification on a simple multiplication of planar measurements corresponding to our dimensions $a \times d \times e$. According to our findings, the results based on this formula are inaccurate without using coefficients in cubic equation (Table 3). Mergo et al. ${ }^{8}$ described volume quantification based on a combination of planar measurements corresponding to our dimensions $a$ and $d$ and recommended the formula $a \times d \times d$ for volume quantification. Based on our results, even when appropriate coefficients in quadratic equation were used, the results had the greatest average absolute variation of $\pm 208.2 \mathrm{~mL}$ (Table 3 ). In addition to the above-mentioned inaccuracies, both methods require measurement in two planes, which is less practical for quick clinical needs than a single measurement on transversal scan.

The limitations of our extended study consist in pleural effusion measurement error caused by several factors. First, the calculated volume of pleural effusion can be inaccurate because of slice thickness and a different effusion area marked on each scan. Second, effusion shape and dimensions can be influenced by size and shape of the thorax (including scoliosis, hyperkyphosis), lung elasticity and the depth of inspiration during scanning. We tried to decrease these inaccuracies by stating the inclusion criteria. A third disadvantage would be the fact that the equation found for dimension $b$ is applicable only for measurements greater than $6 \mathrm{~mm}$. In smaller dimensions the calculated effusion volume has negative values. However, we consider all the above-mentioned limitations nonsignificant.

\section{CONCLUSION}

In conclusion, the described method of pleural effusion volume quantification based on a single planar measurement on CT scan is widely applicable in adult patients with free pleural effusion, easy to perform and appropriately accurate. Table 4 can be easily and effectively used in everyday practice.

\section{ACKNOWLEDGEMENT}

This research was supported by IGA UP Olomouc grant number LF_2012_008.

\section{REFERENCES}

1. Miserocchi G. Physiology and pathophysiology of pleural fluid turnover. Eur Respir J 1997;10:219-25.

2. Lai-Fook SJ. Pleural mechanics and fluid exchange. Physiol Rev 2004;84:385-410.

3. Noppen M, De Waelle M, Li R, Gucht KV, D'Haese J, Gerlo E, Vincken W. Volume and cellular content of normal pleural fluid in humans examined by pleural lavage. Am J Respir Crit Care Med 2000;162:10236.

4. Estenne M, Yernault JC, De Troyer A. Mechanism of relief of dyspnea after thoracocentesis in patients with large pleural effusions. Am J Med 1983;74:813-9.

5. Blackmore CC, Black WC, Dallas RV, Crow HC. Pleural fluid volume estimation: a chest radiograph prediction rule. Acad Radiol 1996;3:1039

6. Eibenberger KL, Dock WI, Ammann ME, Dorffner R, Hörmann MF Grabenwöger F. Quantification of pleural effusions: sonography versus radiography. Radiology 1994;191:681-4.

7. Kalokairinos MI, Vassiliadis SG, Vossou CG, Ciurea AI, Ciortea CA. Estimation of the volume of the pleural effusion by computed tomography. 2010; www.myESR.org - EPOS. doi:10.1594/ ecr2010/C-0902.

8. Mergo PJ, Helmberger T, Didovic J, Cernigliaro J, Ros PR, Staab EV. New formula for quantification of pleural effusions from computed tomography. J Thorac Imaging 1999;14:122-5.

9. Hazlinger M, Heřman M, Čtvrtlík F. Stanovení objemu pleurální tekutiny na CT jednoduchým měřením. Ces Radiol 2010;64:213-7. 